\section{Confidence intervals for frequencies of rare alleles}

Dear Sir,

Chuang et al. [1] have measured the frequencies of a mutation of the amylin/IAPP gene in Taiwanese patients.

Table 1 of their letter lists frequencies with confidence intervals in four groups of subjects. Clearly, a negative gene frequency is not meaningful, so the $95 \%$ (or any other) confidence interval cannot extend to $-0.6 \%$.

The authors have obviously used the large sample approximation for the confidence intervals of a proportion (incorrectly in one case), but they do not have a large sample as far as this mutation is concerned. A more accurate calculation is readily available [2] and applying it to these data gives the following results:

Corresponding author: Dr. P. McShane, Nuffield Dept. of Surgery, John Radcliffe Hospital, Headington, Oxford, OX3 9DU, UK

$\begin{array}{lllll} & \text { Control } & \text { IGT } & \text { Type I } & \text { Type II } \\ \text { Percentage } & 4.04 & 4.16 & 1.64 & 1.64 \\ \text { Interval } & 1.1-10.0 & 1.05-21.2 & 0.2-5.7 & 0.3-4.7\end{array}$

Incorrect confidence intervals could lead to erroneous conclusions when comparing populations.

I have written a macro to do this calculation in Minitab (Minitab Inc., State College, Pa., USA) which I can supply (preferably by e-mail). It would not be difficult to do so for other programs also.

Yours sincerely,

P.McShane

\section{References}

1. Chuang L-M, Lee K-C, Huang C-N, Wu H-P, Tai T-Y, Lin B (1998) Role of S20G mutation of amylin gene in insulin secretion, insulin sensitivity and Type II diabetes mellitus in Taiwanese patients. Diabetologia 41: 1250-1251

2. Armitage P, Berry G (1987) Statistical Methods in Medical Research (2nd ed.) Blackwell Scientific Publishers, Oxford, pp 118-119

\section{Is the newly suggested fasting plasma glucose cut-off point for the diagnosis of diabetes the right one?}

Dear Sir,

Recently the Expert Committee of the American Diabetes Association (ADA) suggested new criteria for the diagnosis of diabetes [1], with lowering of the fasting plasma glucose cut-off from $7.8 \mathrm{mmol} / \mathrm{l}$ to $7.0 \mathrm{mmol} / \mathrm{l}$. This change was based on the predictive power for microvascular disease in cross-sectional trials as well as on the equivalence with the $2 \mathrm{~h}$-plasma glucose value of $11.1 \mathrm{mmol} / \mathrm{l}$ after a $75 \mathrm{~g}$ oral glucose tolerance test (OGTT). The World Health Organisation (WHO) is currently in the process of revising the diabetic diagnostic criteria [2]. Since most of the data taken into consideration by the ADA were derived from non-European populations, i. e. Pima Indians, Egyptians, several Pacific populations, adults from the United States (NHANES) etc., we thought it would be of interest to report about the equivalence of fasting and $2 \mathrm{~h}$-plasma glucose during OGTT in a European population.

Data were evaluated from the RIAD study, a prospective survey on Risk factors in IGT for Atherosclerosis and Diabetes, details of which have been published previously [3]. Briefly, 1139 middle-aged subjects (40-70 years of age) at risk for the development of diabetes were included: positive family history, obesity, or dys/hyperlipoproteinaemia or any of them. All participants underwent an examination programme according to a strict protocol [3]. To minimize the errors of the laboratory analysis special efforts were undertaken in preparation of samples, such as immediate separation of plasma under cooling conditions and subsequent measurement by the hexo-

Corresponding author: C. Koehler, Institute for Clinical Metabolic Research, Technical University Dresden, Fetcher Strasse 74, D-01307 Dresden, Germany kinase method (interassay $\mathrm{CV}=1.5 \%$ ). The patients were examined after an overnight fast of at least $10 \mathrm{~h}$ and a normal diet and physical activity for 3 days prior to the test. The evaluation of the cut-off point was done by the SPSS programme. The regression line was chosen using the method of the least squares.

In our study the $2 \mathrm{~h}$-plasma glucose value of $11.1 \mathrm{mmol} / \mathrm{l}$ was found to correspond to $6.81 \mathrm{mmol} / \mathrm{l}$ fasting plasma glucose with a standard error of $0.14 \mathrm{mmol} / \mathrm{l}$ (Fig. 1). The sensitivity of this cut-off point in diagnosing diabetes $(69.7 \%)$ is almost twice as high as the one of the hitherto applied WHO criterion of $7.8 \mathrm{mmol} / 1 \quad(35.3 \%)$ and slightly higher than that of $7.0 \mathrm{mmol} / \mathrm{l}(63.9 \%)$ newly suggested by the ADA. The specificity of a fasting plasma glucose cut-off value of $6.81 \mathrm{mmol} / 1$ is $91.4 \%$, however, and somewhat lower than both ADA and WHO criteria ( $94.8 \%$ and $99.4 \%$ respectively). Our data are consistent with the reports of other working groups from different countries which found a cut-off point, ranging from 5.3 to $7.1 \mathrm{mmol} / \mathrm{l}[4-6]$.

It was shown by Harris et al. that almost all subjects with a fasting plasma glucose of $7.8 \mathrm{mmol} / \mathrm{l}$ or more display a $2 \mathrm{~h}$-plasma glucose of $11.1 \mathrm{mmol} / \mathrm{l}$ or more after an OGTT, whereas only about a quarter of the subjects with a $2 \mathrm{~h}$-plasma glucose of $11.1 \mathrm{mmol} / \mathrm{l}$ or more and without previously known diabetes have a fasting plasma glucose of $7.8 \mathrm{mmol} / 1$ or more [7]. In addition, postprandial hyperglycaemia is known to precede fasting hyperglycaemia for several years. Therefore, summarizing our findings and other reports we agree with the suggested lowering of the fasting plasma glucose cut-off point, which would reflect a similar degree of hyperglycaemia as the $2 \mathrm{~h}$ plasma glucose value of $11.1 \mathrm{mmol} / \mathrm{l}$. In order to obtain an appropriate classification of subjects with isolated postprandial hyperglycaemia, we would, however, recommend the retention of OGTT in the diabetes diagnosis.

Yours sincerely, C. Koehler, T. Temelkova-Kurktschiev, E. Henkel, F. Schaper, K. Fuecker, M. Hanefeld 